\title{
Áreas prioritarias de conservación en la cuenca del río Segura utilizando los coleópteros acuáticos como indicadores
}

\author{
David Sánchez-Fernández, Pedro Abellán, Josefa Velasco, Andrés Millán \\ Departamento de Ecología e Hidrología, Facultad de Biología, Universidad de Murcia, Campus de \\ Espinardo, 30100, Murcia, España. \\ davidsan@um.es, pabellan@um.es, jvelasco@um.es, acmillan@um.es
}

\section{RESUMEN}

Con este trabajo se pretende identificar las áreas prioritarias de conservación en la Cuenca del Río Segura, utilizando los coleópteros acuáticos como herramienta. Se ha empleado el índice denominado Interés de Conservación (IC) (Millán 1991), que atiende a criterios de riqueza y rareza de especies, y es una modificación del índice Rarity Quality Factor (Eyre \& Rushton, 1989). Este índice se ha aplicado a la comunidad de Adephaga acuáticos y al total de coleópteros acuáticos, tanto por estaciones (248 y 377) como por cuadrículas UTM de 10 x $10 \mathrm{Km}$. (116 cuadrículas, aproximadamente el 50\% de la superficie total). En función de los valores del IC se han clasificado las estaciones y cuadrículas según su interés de conservación y se han identificado las áreas prioritarias de conservación para la Cuenca del Río Segura. Por último, se ha elaborado una cartografía superponiendo las áreas prioritarias y los Espacios Naturales Protegidos, para determinar el grado de solapamiento existente y detectar vacíos en conservación. Los resultados más destacables son:

1.- Se ha registrado un total de 230 especies de coleópteros acuáticos, aproximadamente el 36 \% de la riqueza de la Península Ibérica, de las cuales 32 son endemismos ibéricos, 4 de ellos exclusivos para la Cuenca del Río Segura (Hydraena mecai, H. mandfredjaechi, Limnebius millani y Ochthebius albacetinus).

2.- Dos estaciones, Nacimiento del Río Madera y Chorros del Río Mundo, aparecen siempre dentro de las 10 más valoradas, por lo que merecen una especial atención cara a su conservación.

3. Los Adephaga acuáticos pueden reflejar condiciones ambientales similares al conjunto de los coleópteros acuáticos, constituyendo un buen indicador de biodiversidad.

4. La mayoría de las áreas con interés de conservación máximo se localizan en tramos de cabecera, en el noroeste de la cuenca, con predominio de hábitats tipo como arroyos de montaña.

5. La coincidencia entre las áreas prioritarias de conservación y los ENPs ("Espacios Naturales Protegidos") es de aproximadamente el $67 \%$, por lo tanto, existe un vacío que representa el $33 \%$ de la superficie considerada como de interés de conservación máximo.

Palabras clave: Conservación, Coleópteros Acuáticos, Cuenca del Río Segura, Índices, Rareza, Riqueza.

\begin{abstract}
This work tries to identify the areas with the highest interest for conservation in the Segura River basin using the aquatic coleopterans as a tool. The "Interés de Conservación" (Conservation Interest) index (IC) (Millán, 1991) was used, which regards to species richness and rarity. This index is a modification of the Rarity Quality Factor (Eyre \& Rushton, 1989). This index has been applied to the community of aquatic Adephaga and to all aquatic coleopterans, both using sampled sites (248 and 377 for Adephaga and all Coleoptera respectively) and U.T.M $10 \times 10 \mathrm{~km}$ squares (116 squares, about $50 \%$ of the total basin area). On the basis of the IC values we have classified the sampled localities and squares according to their conservation interest, and we have identified the priority areas for conservation in the Segura River Basin. Lastly, a map has been elaborated of the priority areas determined by this study and the protected areas to detect possible gaps in conservation. The most important results are:

1. A total of 230 species of aquatic Coleoptera have been recorded, about $36 \%$ of the total richness of the Iberian Peninsula. 32 of them are Iberian endemics, and 4 are endemics exclusive of the Segura River Basin (Hydraena mecai, H. mandfredjaechi, Limnebius millani and Ochthebius albacetinus).

2. Two sampled localities, Nacimiento del Río Madera and Chorros del Río Mundo, always appear among the 10 highest scores. These places need more attention for better conservation.
\end{abstract}


3. The aquatic Adephaga were indicators of similar environmental conditions than the total community of aquatic Coleoptera, being a good indicator of total biodiversity.

4. Most of the places with the highest conservation interest are located in the source area of the rivers, in the northwest of the Segura Basin, with mountain streams as a dominant habitat type.

5. The overlapping between high-priority areas for conservation and Protected Natural Spaces("PNSs") was about 67\%, revealing a gap that includes 33\% of the area classified as of highest conservational interest in this work.

Keywords: Conservation, Aquatic Coleoptera, Segura River Basin, Index, Rarity, Richness.

\section{INTRODUCCIÓN}

Para la determinación de las áreas prioritarias de conservación cara a su gestión es de gran utilidad la identificación de espacios particularmente ricos en especies, especies raras o amenazadas, o alguna combinación de estos atributos. Estos enclaves se conocen como áreas críticas de biodiversidad o hotspots (Reid, 1998). Para identificar estas zonas, únicamente se atiende a determinados taxones, generalmente plantas y vertebrados. En muy pocas ocasiones se han tenido en cuenta a los invertebrados, a pesar de representar aproximadamente el 95\% de todas las especies vivientes (Hull et al., 1998; Palmer, 1999; Sluys, 1999).

La cuenca mediterránea es una de las 25 áreas propuestas como hotspots a nivel mundial (Myer et al., 2000), y dentro de ésta, uno de los puntos críticos de biodiversidad más importantes es el Sureste ibérico, tanto por la gran variedad y endemicidad de formas de vida que presenta, como por la significativa transformación antropogénica del medio que desde hace miles de años se viene realizando (Dodson et al., 1998). Por otro lado, se trata de la zona más árida de Europa, por lo que sus ecosistemas están sometidos a un importante estrés hídrico y erosión del suelo (López Bermúdez, 1999).

Aunque en los últimos años se han desarrollado multitud de índices que utilizan los macroinvertebrados acuáticos para determinar la calidad de las aguas continentales (Eyre, 1996), son muy pocos los trabajos que han desarrollado índices específicos que permitan conocer el estado de conservación o grado de naturalidad de dichos ecosistemas acuáticos. Entre ellos destacan los trabajos de Foster (1987) y
Eyre \& Rushton (1989), que utilizan los coleópteros acuáticos como indicadores, al reflejar las mismas condiciones ambientales que la comunidad de macroinvertebrados en su conjunto (Jeffries, 1988).

Los coleópteros acuáticos constituyen una buena herramienta por el gran número de especies que presentan, su gran diversidad ecológico-funcional, la amplia variedad de medios que ocupan y el buen conocimiento taxonómico y faunístico que actualmente se tiene de ellos (Ribera \& Foster, 1993). Además, son importantes descriptores de los cambios espaciales y temporales producidos en los sistemas fluviales (Richoux \& Castella; 1986, Richoux, 1988).

En la península Ibérica, únicamente Millán (1991) utiliza los coleópteros acuáticos como herramienta para valorar la necesidad de conservación de los ecosistemas acuáticos. En dicho trabajo se clasifican 248 estaciones de muestreo de la Cuenca del Río Segura a partir de la aplicación del índice Interés de conservación (IC), utilizando la riqueza y rareza de las especies de Adephaga acuáticos.

Los objetivos del presente trabajo son los siguientes:

1. Identificar la riqueza, rareza y endemicidad de las especies de coleópteros acuáticos en la Cuenca del Río Segura.

2. Determinar si los Adephaga acuáticos son buenos indicadores de las áreas con mayor valor natural aplicando el índice Interés de Conservación (IC).

3. Clasificar las áreas, a partir de cuadrículas UTM de 10 x $10 \mathrm{Km}$., según los valores del IC para reconocer los enclaves prioritarios de conservación. 
4. Detectar vacíos en la red de Espacios Naturales Protegidos (ENPs) de la Cuenca del Segura, a través de la superposición cartográfica de las áreas prioritarias de conservación y los ENPs actualmente reconocidos en dicha cuenca.

\section{MATERIAL Y MÉTODOS}

\section{Área de estudio y recopilación de datos}

El presente estudio se enmarca en el contexto de la Cuenca Hidrográfica del Río Segura, recogiendo los datos de la presencia de especies de coleópteros acuáticos en un total de 377 estaciones de muestreo dentro de la misma.

La superficie prospectada corresponde a 116 cuadrículas UTM (Elipsoide Hayford) de 10 x 10 $\mathrm{Km}$., de las 231 que tiene la Cuenca Hidrográfica del Río Segura (Fig.1), lo que supone aproximadamente, un $50 \%$ de la superficie de la misma, recogiendo la mayor parte de la variabilidad de cuerpos de agua presentes en ella.

El trabajo compila, por un lado, los muestreos llevados a cabo durante los años 1981-2002, aunque de manera más específica para coleópteros acuáticos desde 1994, y por otro, la información obtenida sobre este grupo taxonómico, a través de una exhaustiva recopilación bibliográfica.

Si bien no se puede hablar de un esfuerzo de muestreo similar en todas las estaciones y cuadrículas, debido al largo periodo de años en el que se han obtenido los datos utilizados en este estudio y al diferente origen de los mismos, la mayor parte de ellos proceden de estimas relativas utilizando una manga entomológica, pentagonal o triangular, de entre $250 \mathrm{~mm}$ y $1 \mathrm{~mm}$ de luz de malla y con una profundidad de red de $20-30 \mathrm{~cm}$.

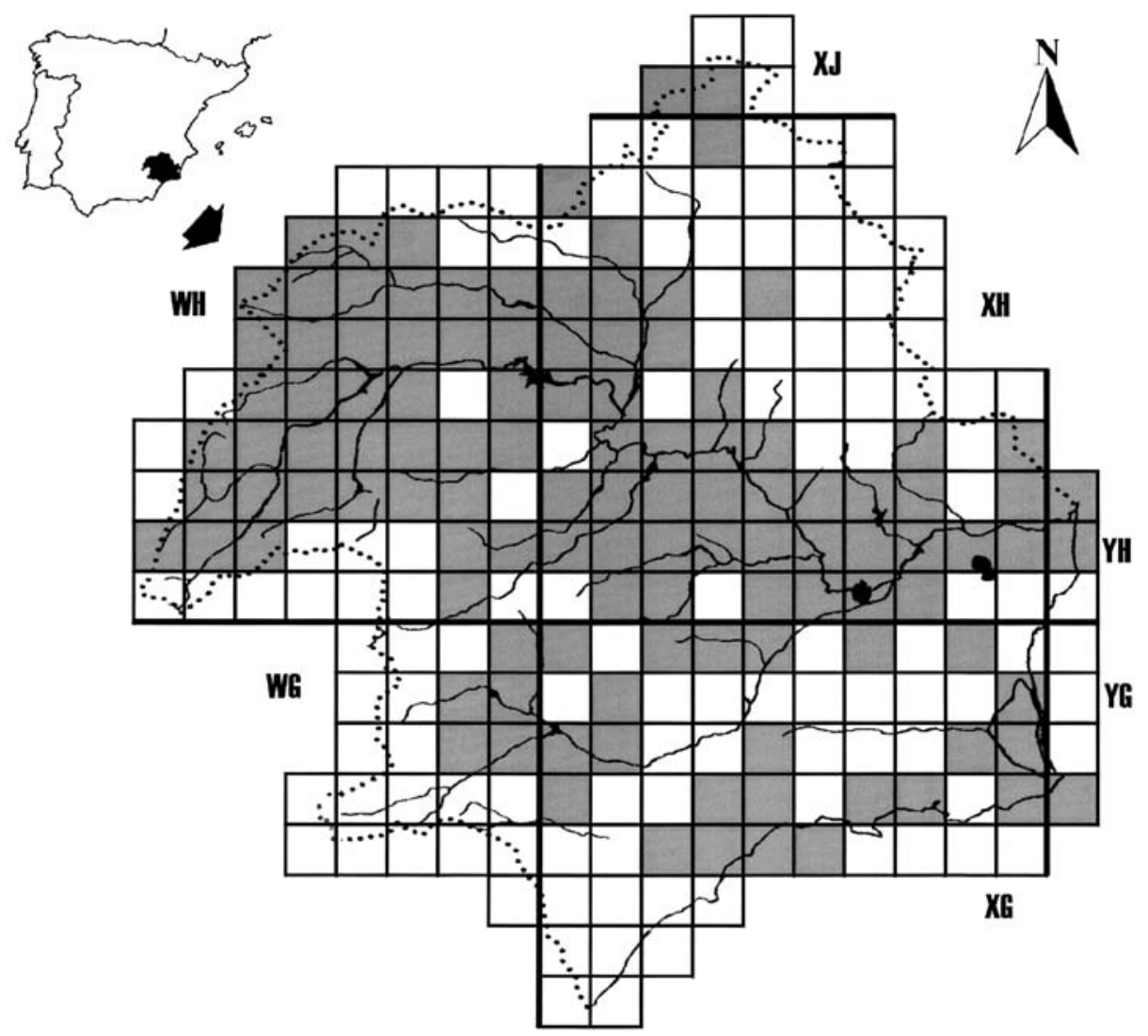

Figura 1. Localización de la Cuenca Hidrográfica del Río Segura y superficie muestreada en cuadrículas UTM 10 x $10 \mathrm{Km}$. Location of Segura River Basin and sampled surface in UTM squares $10 \times 10 \mathrm{Km}$. 
La prospección siempre se ha realizado de manera estratificada, muestreando aquellos hábitats ecológica y fisonómicamente diferentes, dentro de la estación de muestreo y hasta que, aparentemente, dejaran de aparecer nuevas especies.

Para la consideración de especies endémicas en la Península Ibérica se ha seguido, en parte, a Ribera (2000), clasificándolas en:

- No estrictas (NE): aquellas que tienen una distribución que excede, en ciertas zonas del entorno de los Pirineos, los límites ibéricos.

- Generales (G): con un rango de distribución amplio en la Península Ibérica.

- Sur (S): son endemismos que tienen su distribución restringida al Sur, Sureste y, excepcionalmente, Suroeste de la Península Ibérica.

- Exclusivas (E): únicamente encontradas en la Cuenca Hidrográfica del Río Segura.

De igual forma, se han considerado como especies raras para la Cuenca del Río Segura aquellas que aparecen sólo en una cuadrícula UTM 10 x $10 \mathrm{Km}$. dentro del área de estudio.

\section{Aplicación de índices y selección de áreas}

Para determinar las estaciones de muestreo y cuadrículas UTM 10 x $10 \mathrm{Km}$. de mayor interés de conservación en la Cuenca del Río Segura, se ha aplicado a las estaciones y cuadrículas estudiadas el índice denominado Interés de Conservación (IC) (Millán, 1991). Este índice utiliza, conjuntamente, criterios de riqueza y rareza de especies de coleópteros acuáticos y se

Tabla 1. Tipos y valores de rareza para las especies. Tomado de Eyre \& Rushton (1989). Types and rarity values for the species. According to Eyre \& Rushton (1989).

\begin{tabular}{ccc}
\hline $\begin{array}{c}\text { Tipo } \\
\text { de rareza }\end{array}$ & Presencia en... & $\begin{array}{c}\text { Valor } \\
\text { de rareza }\end{array}$ \\
\hline Tipo 1 & $>$ 64 estaciones/cuadrículas & 1 \\
Tipo 2 & 32-64 estaciones/cuadrículas & 2 \\
Tipo 3 & 16-31 estaciones/cuadrículas & 4 \\
Tipo 4 & 8-15 estaciones/cuadrículas & 8 \\
Tipo 5 & 4-7 estaciones/cuadrículas & 16 \\
Tipo 6 & 2-3 estaciones/cuadrículas & 32 \\
Tipo 7 & 1 estación/cuadrícula & 64 \\
\hline
\end{tabular}

trata de una modificación del índice Rarity Quality Factor (RQF) propuesto por Eyre \& Rushton (1989).

Para la aplicación del índice, en primer lugar se han establecido los tipos de rareza de las especies en función del número de veces que aparece cada una de ellas en las estaciones de muestreo o cuadrículas. A cada uno de estos tipos se le asocia un valor según una escala geométrica (Tabla 1).

Seguidamente, el IC para cada estación o cuadrícula se ha calculado del siguiente modo:

a) Se suman los valores de rareza de las especies presentes en cada una de ellas:

$$
\Sigma(\mathrm{VR})
$$

b) Posteriormente, para dar mayor peso a la asociación de especies raras, a este primer valor se le añade un segundo término (VA), que se calcula eliminando los valores de rareza 1 y equiparando los valores de mayor rareza de la estación o cuadrícula a los inmediatamente inferiores presentes en la misma.

c) Con la suma de estos 2 términos se obtiene el valor de la rareza de asociación o Rarity Association Total (RAT):

$$
\mathrm{RAT}=\Sigma(\mathrm{VR})+\mathrm{VA}
$$

d) El valor final del RQF de una estación o cuadrícula se obtiene dividiendo el valor de RAT entre el número de especies presentes en cada estación o cuadrícula $(\mathrm{Nx})$ :

$$
\mathrm{RQF}=\mathrm{RAT} / \mathrm{Nx}
$$

e) El IC, que es el valor final de interés de conservación de las estaciones de muestreo y cuadrículas UTM, se obtiene del cálculo del valor medio del RQF y un nuevo término, denominado Riqueza Asociada a Rareza (RR). Este término supone una modificación del RAT en función de la riqueza relativa, y se calcula multiplicando el valor de RAT por la proporción de especies presentes en cada 
estación o cuadrícula con respecto al total de especies de coleópteros acuáticos registradas en la Cuenca del Río Segura (Nt):

$$
\begin{gathered}
\mathrm{RR}=\mathrm{Nx} / \mathrm{Nt} . \\
\mathrm{IC}=(\mathrm{RQF}+\mathrm{RR}) / 2
\end{gathered}
$$

Millán (1991) utiliza únicamente los Adephaga acuáticos para 248 estaciones de muestreo de la Cuenca del Río Segura (numeradas hasta la 390 siguiendo un código general de identificación). Por ello, en primer lugar se ha aplicado el IC a estas 248 estaciones para los Adephaga acuáticos, con el fin de comparar la selección de las 10 estaciones de mayor valor de conservación aportada por dicho autor en 1991 respecto a la selección actual a partir de los datos acumulados hasta la fecha.

A continuación, se ha aplicado el IC a las 377 estaciones de muestreo contempladas en el presente estudio, que incorpora 129 nuevas estaciones a las 248 prospectadas por Millán (1991) (numeradas desde la 391), considerando los coleópteros del grupo Adephaga acuáticos, por un lado, y la totalidad de coleópteros acuáticos registrados en la cuenca, por otro. $\mathrm{La}$ comparación de la ordenación de las 10 primeras estaciones de mayor interés de conservación aportadas por ambos datos ha permitido determinar el valor indicador de los Adephaga acuáticos con respecto al total de la comunidad de coleópteros acuáticos.

Para definir prioridades de conservación, trabajando con datos de presencia y ausencia de especies, resulta imprescindible expresar los datos en unidades de superficie, aplicables, por ejemplo, a las categorías y criterios de la Unión Internacional para la Conservación de la Naturaleza (IUCN, 1996). Por ello, se ha calculado el IC para el conjunto de las 116 cuadrículas donde se incluyen los datos de las 377 estaciones de muestreo.

Los valores del índice obtenidos para cada cuadrícula han permitido ordenarlas de mayor a menor. A continuación, se ha realizado una clasificación de estos valores en 5 clases en función
Tabla 2. Clasificación de las cuadrículas en 5 niveles según su valor de IC. Classification of the squares in 5 levels according to their IC value of.

\begin{tabular}{lc}
\hline $\begin{array}{l}\text { Valor de conservación máximo } \\
\text { Interés de conservación alto }\end{array}$ & $\mathrm{IC}>75$ \\
Interés de conservación medio & $\mathrm{IC}>21,8$ \\
(quartil superior) & $\mathrm{IC}>10,14$ \\
Interés de conservación bajo & $\mathrm{IC}>4,95$ \\
Interés de conservación nulo & (cuartil inferior) \\
\hline
\end{tabular}

de su interés de conservación (ver Anexo 2). La clase de interés de conservación máximo, recoge las 12 cuadrículas con los valores más altos de IC (>75), claramente separados de los del resto de cuadrículas, las cuales, a su vez, se han dividido en cuatro categorías a partir de los valores del cuartil superior, mediana y cuartil inferior, denominándose de interés de conservación alto, medio, bajo y nulo, respectivamente (Tabla 2).

Por último, se ha elaborado una cartografía de la cuenca con las cuadrículas definidas como áreas prioritarias de conservación y los Espacios Naturales Protegidos (ENPs) de la cuenca, con el fin de determinar la coincidencia entre ambos y la existencia de vacíos de protección en cuanto a ecosistemas acuáticos. Si bien existe cierta disparidad, entre las distintas comunidades autónomas, para designar la figura de protección de cada una de las áreas que componen su red de espacios protegidos, en el presente trabajo se han considerado todas aquellas que las distintas administraciones autonómicas implicadas dentro de los límites de la Cuenca Hidrográfica del Río Segura incluyen en su "red de espacios naturales protegidos".

\section{RESULTADOS}

Se han registrado 230 especies para la Cuenca del Río Segura (Anexo 1), de las cuales 32 son endemismos ibéricos y 4 de ellos endemismos exclusivos para la cuenca (Hydraena mecai, Hydraena manfredjaechi, Limnebius millani y Ochthebius albacetinus). Esta riqueza supone 
Tabla 3. Relación de las 10 estaciones con mayor interés de conservación según su valor de IC obtenido a partir de la presencia de Adephaga acuáticos en 248 estaciones hasta 1991. (Cód: Código de las estaciones de muestreo, S: Riqueza, IC: Interés de Conservación). Ranking of the 10 sites with the highest conservation interest using IC scores obtained from the presence of aquatic Adephaga in 248 sites until 1991. (Cód: Code of the sampling sites, S: richness, IC: value of Conservation Interest).

\begin{tabular}{|c|c|c|c|c|}
\hline $\mathbf{N}^{\mathbf{o}}$ & Cód. & Denominación & $\mathbf{S}$ & IC \\
\hline 1 & 120 & Río Zumeta. Santiago de la Espada & 17 & 48.99 \\
\hline 2 & 108 & Nacimiento del Río Madera & 15 & 41.69 \\
\hline 3 & 113 & Desembocadura del Río Madera & 11 & 37.45 \\
\hline 4 & 384 & Humedales de la Laguna de El Hondo. Balsa de riego & 1 & 32.50 \\
\hline 5 & 144 & Chorros del Río Mundo & 9 & 30.71 \\
\hline 6 & 189 & Balsa próxima al nacimiento del Benamor. Poza & 13 & 30.53 \\
\hline 7 & 386 & Laguna de autodepuración. Espinardo & 11 & 30.09 \\
\hline 8 & 383 & Charca lateral en el Río Madera, camino de Siles & 2 & 29.75 \\
\hline 9 & 109 & Río Madera. Peña Rubia & 13 & 26.46 \\
\hline 10 & 389 & Fuente de La Toba & 2 & 25.50 \\
\hline
\end{tabular}

aproximadamente un $36 \%$ del total de las especies de la Península Ibérica (Millán et al., 2002).

A efectos del cálculo del índice no se han tenido en cuenta 5 de ellas (Enochrus melanocephalus, Paracymus relaxus, Donacia andalusiaca, Donacia aquatica y Plateumaris sericea), al no conocerse la localización exacta de las citas.

De la comparación de las 10 estaciones que, utilizando los Adephaga acuáticos exclusivamente, han alcanzado los valores más altos de IC, con los datos aportados por Millán (1991) (Tablas 3 y 4), se observa que 4 de las estaciones que aparecían en la ordenación realizada en 1991, se mantienen en 2002. Éstas, corresponden a arroyos de montaña: Nacimiento del Río
Madera, Chorros del Río Mundo, Río Zumeta en Santiago de la Espada y Desembocadura del Río Madera. Por otro lado, 2 estaciones que en 1991 no aparecían en la selección, en 2002 presentan los mayores valores de IC, son el Río de la Vega y el Río Endrinales, también arroyos de montaña. Además, aparecen otras 2 nuevas estaciones, que son el Río Quípar en la Encarnación y la Balsa de Tébar. Ambas corresponden a hábitats tipo claramente diferenciados de los arroyos de media montaña, la primera encuadrada dentro de arroyos propios de la vega media y la segunda referida a un ambiente lenítico artificial naturalizado con la presencia de una especie rara, Cybister tripunctatus africanus.

Tabla 4. Relación de las 10 estaciones con mayor interés de conservación según su valor de IC obtenido a partir de la presencia de Adephaga acuáticos en 248 estaciones hasta 2002. (Cód: Código de las estaciones de muestreo, S: Riqueza, IC: Interés de Conservación). Ranking of the 10 sites with the highest conservation interest using IC scores obtained from the presence of aquatic Adephaga in 248 sites until 2002. (Cód: Code of the sampling sites, S: richness, IC: value of Conservation Interest).

\begin{tabular}{|c|c|c|c|c|}
\hline $\mathbf{N}^{\mathbf{o}}$ & Cód. & Denominación & $\mathbf{S}$ & IC \\
\hline 1 & 141 & Río de la Vega & 22 & 35.1 \\
\hline 2 & 163 & Río Endrinales & 18 & 33.8 \\
\hline 3 & 108 & Nacimiento del río Madera & 19 & 28.6 \\
\hline 4 & 144 & Chorros del río Mundo & 13 & 28.3 \\
\hline 5 & 120 & Río Zumeta. Santiago de la Espada & 17 & 28.2 \\
\hline 6 & 113 & Desembocadura del río Madera & 12 & 20.7 \\
\hline 7 & 221 & Río Quípar en La Encarnación & 14 & 18.5 \\
\hline 8 & 369 & Balsa de Tébar & 2 & 17.3 \\
\hline 9 & 385 & Arroyo Cañada Hermosa & 2 & 17.3 \\
\hline 10 & 164 & Río Madera (río Mundo) & 8 & 15.9 \\
\hline
\end{tabular}


Tabla 5. Relación de las 10 estaciones con mayor interés de conservación según su valor de IC obtenido a partir de la presencia de Adephaga acuáticos en 377 estaciones hasta 2002. (Cód: Código de las estaciones de muestreo, S: Riqueza, IC: Interés de Conservación). Ranking of the 10 sites with the highest conservation interest using IC scores obtained from the presence of aquatic Adephaga in 377 sites until 2002. (Cód: Code of the sampling sites, S: richness, IC: value of Conservation Interest).

\begin{tabular}{rllrr}
\hline $\mathbf{N}^{\mathbf{0}}$ & Cód. & \multicolumn{1}{c}{ Denominación } & S & IC \\
\hline 1 & 162 & Río Endrinales en Las Espineras & 28 & 108.20 \\
2 & 393 & Arroyo de Fuenfría & 25 & 69.44 \\
3 & 485 & Laguna de Pétrola & 21 & 48.22 \\
4 & 531 & Laguna del Salobralejo & 17 & 36.76 \\
5 & 141 & Río de la Vega & 22 & 35.09 \\
6 & 163 & Río Endrinales & 18 & 33.81 \\
7 & 108 & Nacimiento del río Madera & 19 & 28.58 \\
8 & 144 & Chorros del río Mundo & 13 & 28.25 \\
9 & 120 & Río Zumeta. Santiago de la Espada & 17 & 28.22 \\
10 & 465 & Laguna de los Patos & 12 & 21.70 \\
\hline
\end{tabular}

Por otro lado, al comparar las 10 primeras estaciones con mayor interés de conservación para los Adephaga acuáticos y para el conjunto de los coleópteros acuáticos (Tablas 5 y 6), se ha observado que 7 de ellas son coincidentes: Río Endrinales en Las Espineras, Arroyo de Fuenfría (estas 2 estaciones aparecen las primeras en ambas selecciones), Laguna de Pétrola, Nacimiento del Río Madera, Río Endrinales, Chorros del Río Mundo y Río de la Vega, en su mayoría arroyos de media montaña. Además de estas 7, cuando se utilizan sólo los Adephaga acuáticos aparecen la Laguna de Salobralejo, Río Zumeta en Santiago de la Espada y la Laguna de los Patos, incorporando hábitats de carácter lenítico. Para el conjunto de coleópteros las 3 nuevas estaciones son el Río Segura después de la desembocadura del Madera, el Arroyo de la Sierra en los Vados del Tus y el Arroyo Bravo, todos arroyos de montaña.

Los mapas de riqueza, endemicidad y rareza para cada una de las cuadrículas muestreadas se encuentran representados en las figuras 2,3 y 4 respectivamente. En todos ellos se observa que los valores más altos aparecen en el noroeste de la cuenca, entre las Sierras de Cazorla, Segura y las Villas y la Sierra de Alcaraz, aunque también se aprecian datos de rareza y endemicidad importantes en las subcuencas más áridas situadas en la vega media del Segura y en la costa murciana.

Tabla 6.- Relación de las 10 estaciones con mayor interés de conservación según su valor de IC obtenido a partir de la presencia de coleópteros acuáticos en 377 estaciones hasta 2002. (Cód: Código de las estaciones de muestreo, S: Riqueza, IC: Interés de Conservación). Ranking of the 10 sites with the highest conservation interest using IC scores obtained from the presence of aquatic beetles in 377 sites until 2002. (Cód: Code of the sampling sites

\begin{tabular}{|c|c|c|c|c|}
\hline $\mathbf{N}^{\mathbf{o}}$ & Cód. & Denominación & $\mathbf{S}$ & IC \\
\hline 1 & 393 & Arroyo de Fuenfría & 75 & 296.60 \\
\hline 2 & 162 & Río Endrinales en Las Espineras & 65 & 192.50 \\
\hline 3 & 108 & Nacimiento del río Madera & 56 & 114.40 \\
\hline 4 & 144 & Chorros del río Mundo & 43 & 91.90 \\
\hline 5 & 141 & Río de la Vega & 55 & 88.21 \\
\hline 6 & 163 & Río Endrinales & 44 & 79.55 \\
\hline 7 & 3 & Río Segura despues desembocadura del Madera & 42 & 68.04 \\
\hline 8 & 485 & Laguna de Pétrola & 41 & 62.44 \\
\hline 9 & 127 & Arroyo de la Sierra. Vados del Tus & 49 & 57.52 \\
\hline 10 & 544 & Arroyo Bravo & 36 & 57.11 \\
\hline
\end{tabular}




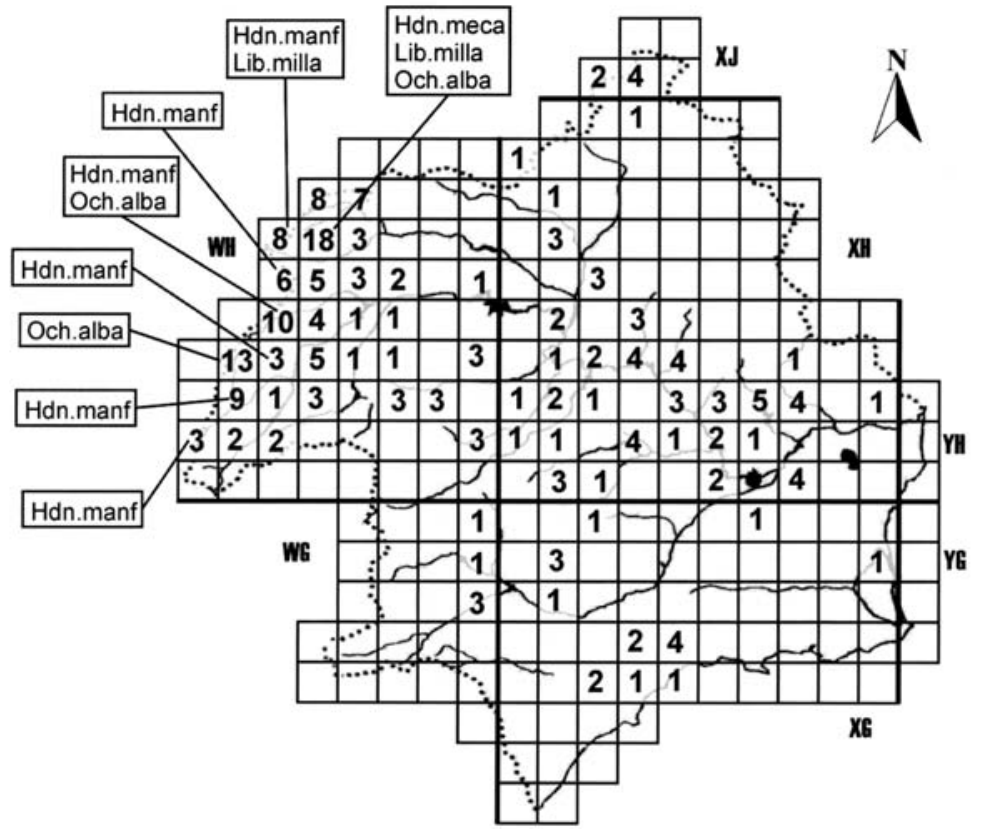

Figura 2. Riqueza de especies por cuadrículas UTM 10 x $10 \mathrm{Km}$. Species richness in UTM $10 \times 10 \mathrm{Km}$ squares.

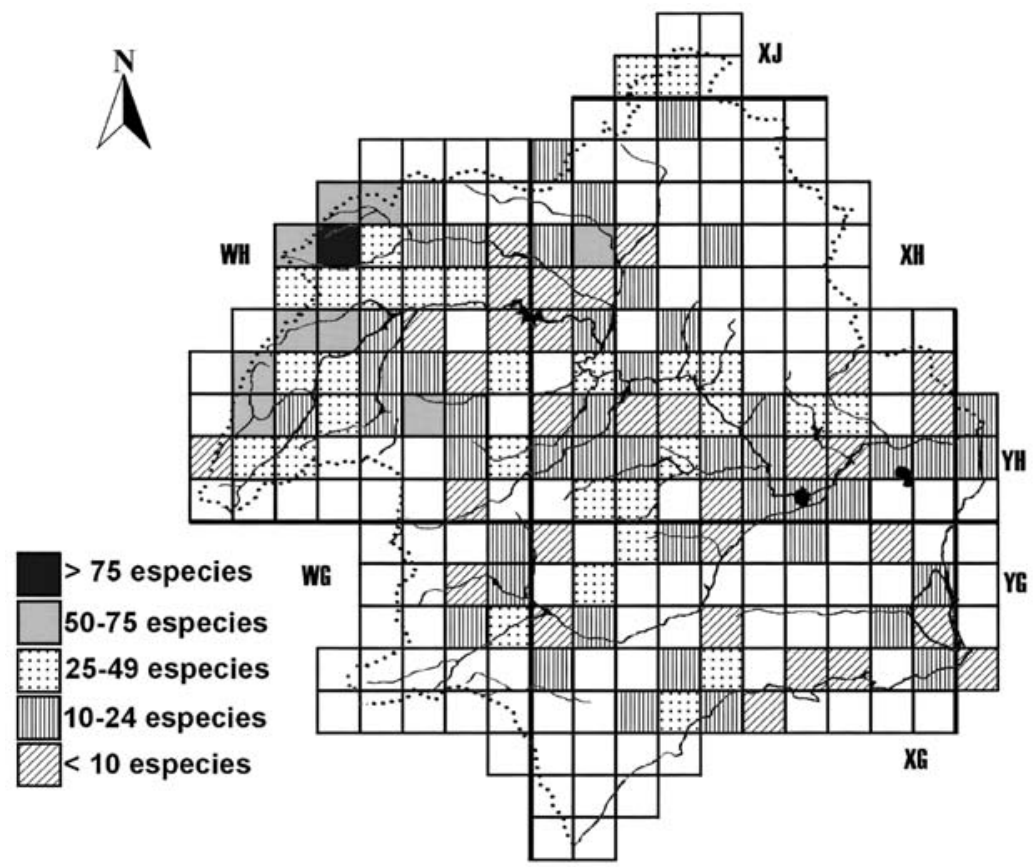

Figura 3. Número de endemismos por cuadrícula UTM 10 x $10 \mathrm{Km}$, destacando la presencia de los endemismos exclusivos (ver Anexo 1 para los códigos de las especies). Number of endemic species in UTM $10 \times 10 \mathrm{Km}$. square, with emphasis on the presence of exclusive endemic species (see Annex 1 for the codes of the species). 
En la figura 5 aparece la clasificación de las cuadrículas UTM 10 × $10 \mathrm{Km}$ de la Cuenca del Río Segura en 5 grupos en función del valor del IC (máximo, alto, medio, bajo y nulo), de las cuales destacan ampliamente las cuadrículas WH5.6, WH3.3 y WH4.6 (ver Anexo 2), que recogen una gran heterogeneidad ambiental, sobresaliendo los arroyos de montaña.

$\mathrm{Al}$ representar en un mismo mapa de la cuenca las 12 cuadrículas seleccionadas como áreas prioritarias de conservación y los espacios naturales protegidos (Fig. 6), se observa que, al menos en parte, 8 de ellas coinciden con algún ENP. El resto está fuera de cualquier tipo de protección, aunque alguna de ellas, como la que incluye la cabecera del Río Benamor, está propuesta como Lugar de Importancia Comunitaria (LIC) para su incorporación en la Red Natura 2000.

\section{DISCUSIÓN}

Durante el periodo de, aproximadamente, veinte años que abarca este estudio, son múltiples los factores que han podido intervenir afectando la composición faunística de las estaciones estudiadas, y por tanto, su ubicación dentro de las clases de interés de conservación propuestas. Esta variación puede ser debida a cambios sucesionales provocados por factores de tipo biótico, cambios abióticos que han afectado a la comunidad derivados de perturbaciones naturales o antropogénicas, o simplemente, cambios en la comunidad ocasionados por el azar o diferencias en la intensidad y frecuencia de muestreo. En cualquier caso, si se comparan los resultados de los cuatro criterios utilizados (ver tablas 3 a 6), dos estaciones, Nacimiento del Río Madera y Chorros del Río Mundo, aparecen siempre dentro de las 10 más valoradas, por lo que merecen una especial atención de cara a su conservación.

En su conjunto se puede destacar que se mantienen los arroyos de montaña como los hábitats tipo con mayor interés de conservación, aspecto observado también por otros autores para el conjunto de la Península Ibérica (Ribera, 2000).

Aunque siempre se ha de tener presente que la información que se obtiene de la aplicación

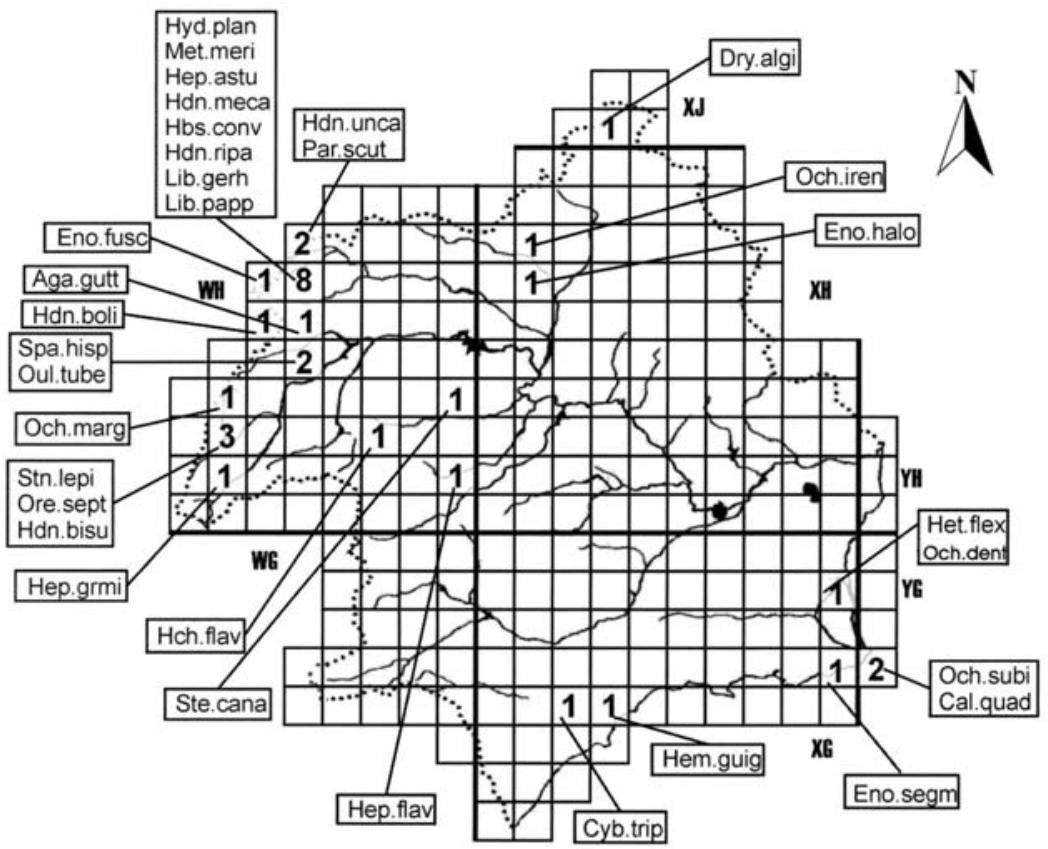

Figura 4. Número de especies raras por cuadrícula UTM 10 x $10 \mathrm{Km}$. (ver Anexo 1 para los códigos de las especies). Number of rare species in square UTM $10 \times 10 \mathrm{Km}$. (see Annex 1 for the codes of the species). 
de cualquier índice con el conjunto de los coleópteros acuáticos es más completa que cuando se aplica utilizando cualquier agrupación taxonómica de éstos, también se ha de considerar la dificultad que entraña conocer la riqueza total de este orden. De este modo, observando los resultados que aparecen en las tablas 5 y 6 , donde 7 estaciones son coincidentes, se puede deducir que los coleópteros Adephaga acuáticos por sí solos, pueden reflejar, de cierta forma, las mismas condiciones que el conjunto de los coleópteros acuáticos, constituyendo un buen indicador de biodiversidad. La mayor diferencia estriba, quizá, en la incorporación de algunos ambientes leníticos cuando se utilizan exclusivamente Adephaga acuáticos, mientras que con todas las familias de coleópteros, el predominio es casi exclusivo de los arroyos de montaña.

Sin embargo, en esta clasificación, quedan fuera de la clase interés máximo de conservación estaciones de gran singularidad pertene- cientes al hábitat tipo ramblas hipersalinas (Millán et al., 1996; Moreno et al., 1997) que, aunque encuadradas dentro de valores altos de conservación, presentan un gran número de especies capaces de colonizar la mayoría de cuerpos de agua con un grado de mineralización moderada. Esto puede deberse al tipo de índice aplicado que prima la riqueza y rareza por encima del tipismo o singularidad del hábitat prospectado. En este sentido, la posible incorporación de este criterio al IC en futuros trabajos sería plausible.

Las cuadrículas con valores más altos del IC $\mathrm{y}$, por tanto, aquellas con mayor interés de conservación aparecen en la figura 6. Dichas cuadrículas incluyen las 10 estaciones de muestreo destacadas cuando se utiliza el total de coleópteros, quedando fuera únicamente 2 estaciones cuando sólo se tienen en cuenta los Adephaga, éstas son la Laguna del Salobralejo (XJ3.0), que se encuentra justo en el límite entre las cla-

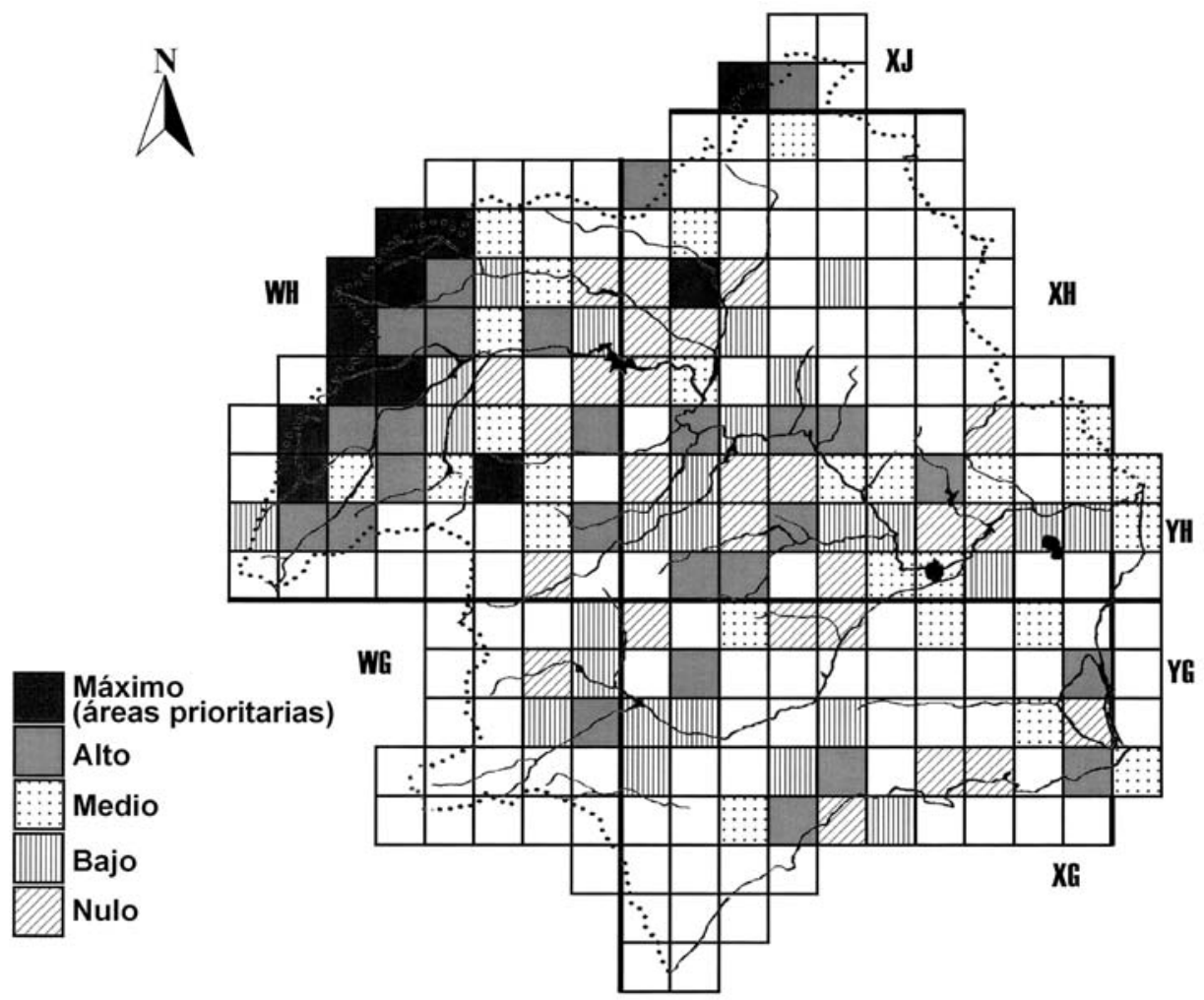

Figura 5. Clasificación de las cuadrículas UTM 10 x $10 \mathrm{Km}$ en función de su interés de conservación. UTM $10 x 10 \mathrm{Km}$. squares classification as a function of their conservation interest. 


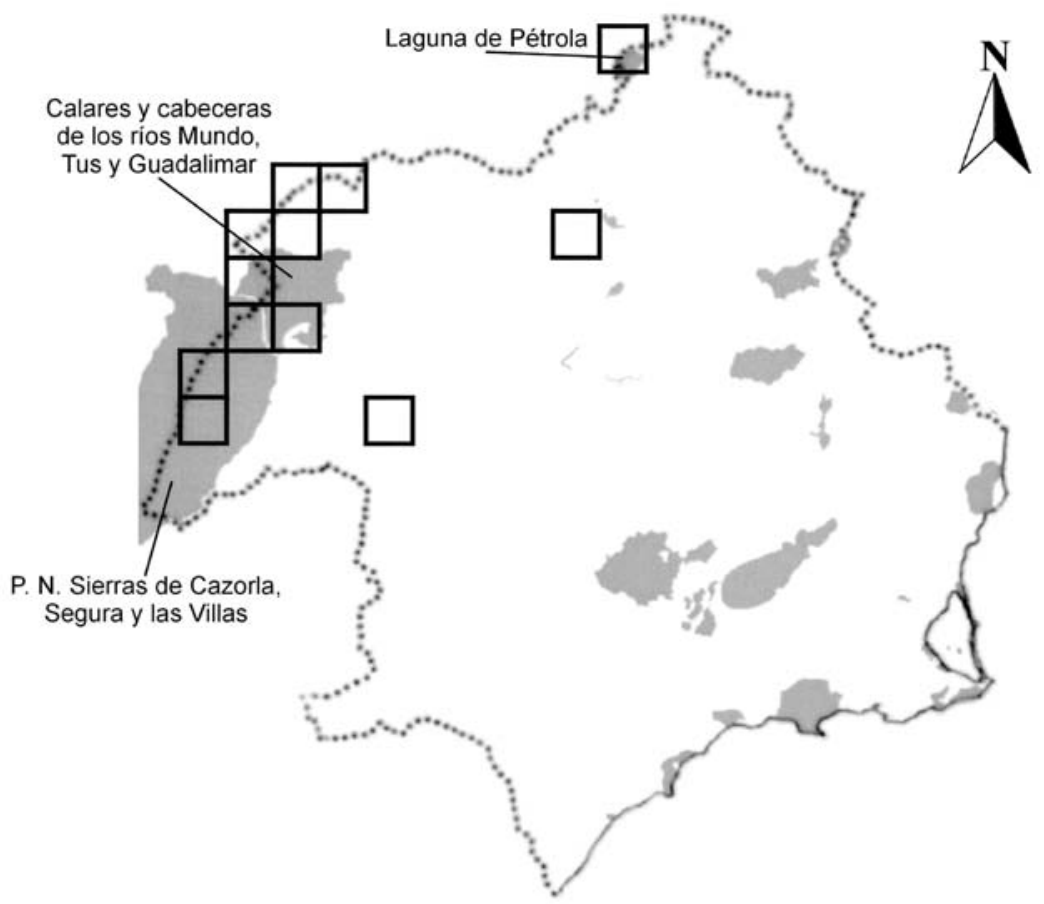

Figura 6. Superposición de áreas prioritarias con mayor valor de conservación definidas en este estudio y Espacios Naturales Protegidos (superficies oscuras) en la cuenca del río Segura. Overlapping of the high-priority areas for conservation and the Natural Protected Areas (dark areas) in the Segura River basin.

ses interés de conservación máximo y alto (Anexo 2) y el Río Zumeta en Santiago de la Espada (WH3.1), perteneciente a la clase de interés de conservación alto.

La mayoría de las 12 cuadrículas con interés de conservación máximo se localizan en tramos de cabecera, en el Noroeste de la cuenca, por lo que predominan los hábitats tipo arroyos de montaña, aunque también aparecen fuentes, lagunas endorreicas, salinas interiores, balsas de riego y estanques artificiales. Esta alta heterogeneidad ambiental posibilita que dichas áreas alberguen 184 de las 230 especies de la cuenca, el $80 \%$ del total, además del $61 \%$ de las especies raras (20 de 33$)$ y el $78 \%$ de las endémicas ( 25 de 32 ), incluyendo las 4 especies endémicas exclusivas para la zona de estudio, por lo que la conservación de estas 12 áreas supondría la protección de la mayoría de especies de coleópteros acuáticos presentes en la Cuenca del Río Segura.
Dentro de estas áreas, la cuadrícula WH5.6, situada en la Sierra de Alcaraz, destaca por encima del resto, pues en las 11 estaciones de muestreo que recoge aparecen 122 especies, de las cuales 18 son endémicas y 8 raras para la Cuenca del Río Segura. Sin embargo, en este caso la heterogeneidad ambiental de la cuadrícula no es alta, ya que la mayoría de las estaciones pertenecen al hábitat tipo arroyos de cabecera o fuentes. Este elevado interés de conservación debe atribuirse a su alto grado de naturalidad. Dentro de esta cuadrícula se encuentran 3 estaciones de muestreo que aparecen en la selección de las 10 primeras utilizando valores del IC para el conjunto de los coleópteros acuáticos (Arroyo de Fuenfría, Río Endrinales en las Espineras y Río Endrinales). Una sola estación de muestreo, el Arroyo de Fuenfría, presenta 11 endemismos ibéricos (Millán \& Aguilera, 2000). Recientemente se ha podido observar ciertas alteraciones antropogénicas en esta estación, principalmente 
debidas a impactos provocados por la actividad turística y recreativa. Actualmente está en marcha el Plan de Ordenación de los Recursos Naturales (PORN) de los Calares y Cabeceras de los Ríos Mundo, Tús y Guadalimar; este espacio se solapa, al menos parcialmente, con 5 de las áreas prioritarias de conservación propuestas en el presente estudio. Aún así, la estación de Fuenfría quedaría fuera de los límites de este Espacio Natural, por lo que se recomienda una ampliación de la superficie del mismo que haga posible su inclusión.

También merece especial atención la cuadrícula XJ2.0, al norte de la Cuenca del Segura, que aparece dentro de las áreas prioritarias de conservación, a pesar de presentar una sola estación de muestreo, la Laguna de Pétrola. Las razones de esta situación pueden ser el marcado gradiente de salinidad, junto con la alta heterogeneidad ambiental que presenta, al poder diferenciarse 4 hábitats distintos (Millán et al., 1996; 2002) que son arroyos de vega media, lagunas endorreicas, pozas y charcas, y salinas interiores. En este sentido, merece la pena destacar el enorme interés de conservación que tienen áreas que reúnen ambientes salinos y de agua dulce en aguas corrientes y estancadas (Millán et al., 2001).

La coincidencia entre las áreas prioritarias de conservación seleccionadas a través del estudio de los coleópteros acuáticos y los ENPs es de aproximadamente el $67 \%$, por lo tanto, existe un vacío que representa el $33 \%$ de la superficie considerada como de interés de conservación máximo, que no presenta ninguna figura de protección. Estas cuadrículas (XH1.6, WH7.2, WH6.7 y WH 5.7, ver figura 6) cuentan con enclaves tan interesantes como la Rambla de la Sierra en Hellín, la Rambla del Ojuelo en Cordovilla, los arroyos de la cuenca del Río Madera, el Río Bogarra y los ríos Alharabe y Benamor en sus zonas de cabecera, por lo que se deberían de tener en cuenta por los gestores para incorporarlas dentro de los límites de los espacios naturales actuales o futuros, o como lugares de importancia comunitaria dentro de la propuesta de la Red Natura 2000.

\section{BIBLIOGRAFÍA}

DODSON, S. L., S. I. ALLEN, F. H. CARPENTER, S. R. IVES, A. R. JEANNE, R. L. KITCHELL, J. F. LANGSTON \& N. E. TURNER. 1998. Ecology. Oxford University Press, New York.

EYRE, M. 1996. A Bibliography of Work Relating to Invertebrates in Environmental Monitoring and Conservation. EMS Workshops. Newcastle upon Tyne.

EYRE, M. D. \& S. P. RUSHTON. 1989. Quantification of conservation criteria using invertebrates. J. Appl. Ecol., 26: 159-71.

FOSTER, G. 1987. Beetles as indicators of wetland conservation quality. In: Environmental and Conservation Using Invertebrates. M.D. Eyre (ed.): 33-35. EMS Publications. Newcastle.

HULL, H. E., S. FREITAG, S. L. CHOWN \& C. L. BELLAMY. 1998. Identification and evaluation of priority conservation areas for Buprestidae (Coleoptera) in South Africa, Lesotho, Swaziland and Namibia. African Entomology, 6: 265-274.

JEFFRIES, M. 1988. Do water beetles comunities reflect the wider freshwater community? The Balfour-Browne Club. Newsletter, 42: 14-17.

IUCN. 1996. IUCN Red List of Threatened Animals. IUCN, Gland, Switzerland.

LÓPEZ BERMÚDEZ, F. 1999. Diversidad y valor de los paisajes secos mediterráneos. En: LibroHomehaje al Prof. Angel Ramos (1926-1998): 711-728. Real Academia de Ciencias Exactas, Físicas y Naturales; E.T.S. de Ingenieros de Montes y Universidad Politécnica de Madrid, Madrid.

MILLÁN, A. 1991. Los Coleópteros Hydradephaga (Haliplidae, Gyrinidae, Noteridae y Dytiscidae) de la cuenca del río Segura (SE de la península Ibérica). Tesis doctoral. Universidad de Murcia. $567 \mathrm{pp}$

MILLÁN, A., J. VELASCO, M. R. VIDAL-ABARCA, M.L. SUÁREZ y L. RAMÍREZ-DÍAZ. 1996. Distribución espacial de los Adephaga acuáticos (Coleoptera) en la cuenca del río Segura (SE de la Península Ibérica). Limnetica, 12(2): 13-29.

MILLÁN, A. \& P. AGUILERA. 2000. A new species of Hydraena Kugelann from the spring of Fuenfría (Segura basin, SE Spain), a site of special conservation interest (Coleoptera: Hydraenidae). Koleopt. Rundsch., 70: 61-64.

MILLÁN, A., J. L. MORENO y J. VELASCO. 2001. Estudio faunístico y ecológico de los coleópteros 
y heterópteros acuáticos de las lagunas de Albacete (Alboraj, Los Patos, Ojos de Villaverde, Ontalafia y Pétrola). Sabuco, 1: 43-94.

MILLÁN, A., J. L. MORENO y J. VELASCO. 2002. Los coleópteros y heterópteros acuáticos y semiacuáticos de la provincia de Albacete. Catálogo faunístico y estudio ecológico. Instituto de Estudios Albacetenses. Albacete.

MORENO, J. L., A. MILLÁN, M. L. SUÁREZ, M. R. VIDAL-ABARCA \& J. VELASCO. 1997. Aquatic Coleoptera and Heteroptera assemblages in waterbodies from ephemeral coastal streams ("ramblas") of south-eastern Spain. Arch. Hydrobiol., 141: 93-107.

MYER, N., R. A. MITTERMEIER, C. G. MITTERMEIER, G. A. B. DA FONSECA \& J. KENT. 2000. Biodiversity hotspots for conservation priorities. Nature, 403: 853-858.

PALMER, M. A. 1999. The application of biogeographical zonation and biodiversity assessment to the conservation of freshwater habitats in Great
Britain. Aquatic Conservation-Marine and Freshwater Ecosystems, 9: 179-208.

REID, W.V. 1998. Biodiversity hotspots. Trends in Ecology and Evolution, 13: 275-280.

RIBERA, I. 2000. Biogeography and conservation of Iberian water beetles. Biol. Conserv., 92: 131-150.

RIBERA, I. \& G. FOSTER. 1993. Uso de Coleópteros acuáticos como indicadores biológicos (Coleoptera). Elitron, 6: 61-75.

RICHOUX, P \& E. CASTELLA. 1986. The aquatic Coleoptera of former riverbeds submitted to large hydrological fluctuations. Proc. $3^{\text {rd }}$ Euopean Cong. Entomol. (Amsterdam, 24-25 August): 129-132.

RICHOUX, P. 1988. Inventaire des Coléoptères des cours d'eau: comparaison de méthodes de prélèvement. Naturaliste Can., 115: 223-228.

SLUYS, R. 1999. Global diversity of land planarians (Platyhelminthes, Tricladida, Terricola): a new indicator-taxon in biodiversity and conservation studies. Biodiversity and Conservation, 8: 1663 1681. 
Anexo 1. Lista de especies de coleópteros acuáticos de la Cuenca Hidrográfica del río Segura (TE: tipo de endemismo, NE: No estricto, G: General, S: Sur, E: Exclusivo; TR(e): tipo de rareza para estaciones; TR(c): Tipo de rareza para cuadrículas. * Especies recogidas en la bibliografía sin especificación de cuadrícula UTM o localidad concreta. ** La presencia de la especie en la Cuenca del Río Segura necesita confirmación. List of aquatic coleopterans of the Segura River Basin (TE: type of endemism, NE: Non strict, G: General, S: South, E: Exclusive; TR (e): type of rarity for the sites; TR(c): type of rarity for square. * Species presented in the bibliography without any specification of U.T.M. square or specific location. ** The presence of the species in the Segura River Basin needs confirmation.

\begin{tabular}{|c|c|c|c|c|c|}
\hline $\mathbf{N}^{\mathbf{o}}$ & Código & Especie & TE & TR(e) & $\operatorname{TR}(\mathrm{c})$ \\
\hline 1 & Spa.hisp & Sphaerius hispanicus (Matthews, 1899) & & 7 & 7 \\
\hline 2 & Gyr.casp & Gyrinus caspius Ménétries, 1832 & & 5 & 5 \\
\hline 3 & Gyr.deje & Gyrinus dejeani Brullé, 1832 & & 3 & 3 \\
\hline 4 & Gyr.dist & Gyrinus distinctus Aubé, 1836 & & 5 & 5 \\
\hline 5 & Gyr.urin & Gyrinus urinator Illiger, 1807 & & 3 & 3 \\
\hline 6 & Aul.stri & Aulonogyrus striatus (Fabricius, 1792) & & 2 & 3 \\
\hline 7 & Orc.vill & Orectochilus villosus (Müller, 1776) & & 2 & 3 \\
\hline 8 & Pel.rotu & Peltodytes rotundatus (Aubé, 1836) & & 4 & 4 \\
\hline 9 & Hal.obli & Haliplus obliquus (Fabricius, 1787) & & 6 & 6 \\
\hline 10 & Hal.line & Haliplus lineatocollis (Marsham, 1802) & & 1 & 1 \\
\hline 11 & Hal.mucr & Haliplus mucronatus Stephens, 1832 & & 1 & 2 \\
\hline 12 & Not.laev & Noterus laevis Sturm, 1834 & & 3 & 4 \\
\hline 13 & Hyb.herm & Hygrobia hermanni (Fabricius, 1775) & & 6 & 6 \\
\hline 14 & Lap.hyal & Laccophilus hyalinus (De Geer, 1774) & & 1 & 1 \\
\hline 15 & Lap.minu & Laccophilus minutus (Linnaeus, 1758) & & 2 & 3 \\
\hline 16 & Lap.poec & Laccophilus poecilus Klug, 1882 & & 6 & 6 \\
\hline 17 & Hyp.aube & Hyphydrus aubei Ganglbauer, 1892 & & 3 & 4 \\
\hline 18 & Hyv.cusp & Hydrovatus cuspidatus (Kunze, 1818) & & 5 & 5 \\
\hline 19 & Yol.bica & Yola bicarinata (Latreille, 1804) & & 2 & 2 \\
\hline 20 & Bid.minu & Bidessus minutissimus (Germar, 1824) & & 2 & 2 \\
\hline 21 & Bid.pumi & Bidessus pumilus (Aubé, 1836) & & 6 & 6 \\
\hline 22 & Hyl.gemi & Hydroglyphus geminus (Fabricius, 1792) & & 1 & 2 \\
\hline 23 & Hyl.sign & Hydroglyphus signatellus (Klug, 1834) & & 3 & 3 \\
\hline 24 & Hyt.conf & Hygrotus confluens (Fabricius, 1787) & & 3 & 4 \\
\hline 25 & Hyt.impr & Hygrotus impressopunctatus (Schaller, 1783) & & 4 & 5 \\
\hline 26 & Hyt.laga & Hygrotus lagari (Fery, 1992) & & 5 & 5 \\
\hline 27 & Hyt.pall & Hygrotus pallidulus (Aubé, 1850) & & 5 & 5 \\
\hline 28 & Her.musi & Herophydrus musicus (Klug, 1833) & & 2 & 3 \\
\hline 29 & Hyd.deci & Hydroporus decipiens Sharp, 1878 & $\mathrm{~S}$ & 6 & 6 \\
\hline 30 & Hyd.disc & Hydroporus discretus Fairmaire, 1859 & & 2 & 3 \\
\hline 31 & Hyd.limb & Hydroporus limbatus Aubé, 1836 & & 4 & 5 \\
\hline 32 & Hyd.luca & Hydroporus lucasi Reiche, 1866 & & 4 & 4 \\
\hline 33 & Hyd.marg & Hydroporus marginatus (Duftschmid, 1805) & & 4 & 5 \\
\hline 34 & Hyd.nigr & Hydroporus nigrita (Fabricius, 1792) & & 5 & 6 \\
\hline 35 & Hyd.norm & Hydroporus normandi Régimbart, 1903 & & 5 & 5 \\
\hline 36 & Hyd.plan & Hydroporus planus (Fabricius, 1781) & & 6 & 7 \\
\hline 37 & Hyd.pube & Hydroporus pubescens (Gyllenhal, 1808) & & 4 & 5 \\
\hline 38 & Hyd.tess & Hydroporus tessellatus Drapiez, 1819 & & 3 & 4 \\
\hline 39 & Grt.aequ & Graptodytes aequalis Zimmermann, 1918 & & 5 & 6 \\
\hline 40 & Grt.cast & Graptodytes castilianus Fery, 1995 & $\mathrm{G}$ & 6 & 6 \\
\hline 41 & Grt.frac & Graptodytes fractus (Sharp, 1880-82) & & 3 & 4 \\
\hline 42 & Grt.igno & Graptodytes ignotus (Mulsant, 1861) & & 4 & 5 \\
\hline 43 & Grt.vari & Graptodytes varius (Aubé, 1836) & & 3 & 4 \\
\hline 44 & Met.meri & Metaporus meridionalis (Aubé, 1836) & & 7 & 7 \\
\hline 45 & Stn.epip & Stictonectes epipleuricus (Seidlitz, 1887) & $\mathrm{NE}$ & 3 & 3 \\
\hline 46 & Stn.lepi & Stictonectes lepidus (Olivier, 1795) & & 7 & 7 \\
\hline 47 & Stn.opta & Stictonectes optatus (Seidlitz, 1887) & & 3 & 3 \\
\hline
\end{tabular}


Anexo 1. (Continuación.)

\begin{tabular}{|c|c|c|c|c|c|}
\hline 48 & Der.depr & Deronectes depressicollis (Rosenhauer, 1856) & $\mathrm{S}$ & 5 & 5 \\
\hline 49 & Der.fair & Deronectes fairmairei (Leprieur, 1876) & & 4 & 4 \\
\hline 50 & Der.hisp & Deronectes hispanicus (Rosenhauer, 1856) & & 4 & 4 \\
\hline 51 & Der.moes & Deronectes moestus (Fairmaire, 1858) & & 3 & 4 \\
\hline 52 & Stt.duod & Stictotarsus duodecimpustulatus (Fabricius, 1792) & & 6 & 6 \\
\hline 53 & Stt.gris & Stictotarsus griseostriatus (De Geer, 1774) & & 6 & 6 \\
\hline 54 & Neb.buch & $\begin{array}{l}\text { Nebrioporus bucheti cazorlensis (Lagar, } \\
\text { Fresneda \& Hernando, 1987) }\end{array}$ & & 3 & 4 \\
\hline 55 & Neb.clar & Nebrioporus clarkii (Wollaston, 1862) & & 2 & 2 \\
\hline 56 & Neb.baet & Nebrioporus baeticus (Schaum, 1864) & $\mathrm{G}$ & 3 & 3 \\
\hline 57 & Neb.ceri & Nebrioporus ceresyi (Aubé, 1836) & & 4 & 4 \\
\hline 58 & Ore.davi & Oreodytes davisii (Curtis, 1831) & & 6 & 6 \\
\hline 59 & Ore.sept & Oreodytes septentrionalis (Gyllenhal, 1827) & & 6 & 7 \\
\hline 60 & Aga.bigu & Agabus biguttatus (Olivier, 1795) & & 3 & 3 \\
\hline 61 & Aga.bipu & Agabus bipustulatus (Linnaeus, 1767) & & 3 & 3 \\
\hline 62 & Aga.brun & Agabus brunneus (Fabricius, 1798) & & 3 & 4 \\
\hline 63 & Aga.cons & Agabus conspersus (Marsham, 1802) & & 5 & 5 \\
\hline 64 & Aga.didy & Agabus didymus (Olivier, 1795) & & 3 & 3 \\
\hline 65 & Aga.gutt & Agabus guttatus (Paykull, 1798) & & 7 & 7 \\
\hline 66 & Aga.nebu & Agabus nebulosus (Forster, 1771) & & 4 & 4 \\
\hline 67 & Aga.niti & Agabus nitidus (Fabricius, 1801) & & 4 & 5 \\
\hline 68 & Aga.palu & Agabus paludosus (Fabricius, 1801) & & 4 & 5 \\
\hline 69 & Aga.ramb & Agabus ramblae Millán \& Ribera, 2001 & $\mathrm{G}$ & 2 & 3 \\
\hline 70 & Ily.chal & Ilybius chalconatus (Panzer, 1796) & & 5 & 5 \\
\hline 71 & Ily.mont & Ilybius montanus (Stephens, 1828) & & 6 & 6 \\
\hline 72 & Ily.meri & Ilybius meridionalis Aubé, 1836 & & 4 & 5 \\
\hline 73 & Rha.sutu & Rhantus suturalis (McLeay, 1825) & & 2 & 3 \\
\hline 74 & Col.fusc & Colymbetes fuscus (Linnaeus, 1758) & & 5 & 6 \\
\hline 75 & Mel.cori & Meladema coriacea Castelnau, 1834 & & 2 & 3 \\
\hline 76 & Ere.gris & Eretes griseus (Fabricius, 1781) & & 3 & 4 \\
\hline 77 & Hyc.lean & Hydaticus leander (Rossi, 1790) & & 3 & 3 \\
\hline 78 & Dyt.cirf & Dytiscus circumflexus Fabricius, 1801 & & 4 & 4 \\
\hline 79 & Dyt.pisa & Dytiscus pisanus Castelnau, 1834 & & 5 & 5 \\
\hline 80 & Cyb.trip & Cybister tripunctatus africanus Castelnau, 1834 & & 7 & 7 \\
\hline 81 & Cyb.late & Cybister lateralimarginalis (De Geer, 1774) & & 4 & 4 \\
\hline 82 & Hep.nubi & Helophorus nubilus Fabricius, 1776 & & 6 & 6 \\
\hline 83 & Hep.alte & Helophorus alternans Gené, 1836 & & 4 & 4 \\
\hline 84 & Hep.mari & Helophorus gr. maritimus Rey, 1885** & & 4 & 5 \\
\hline 85 & Hep.brev & Helophorus brevipalpis Bedel, 1881 & & 4 & 4 \\
\hline 86 & Hep.astu & Helophorus asturiensis Kuwert, 1885 & & 7 & 7 \\
\hline 87 & Hep.grmi & Helophorus gr. minutus Fabricius, 1775 & & 7 & 7 \\
\hline 88 & Hep.flav & Helophorus flavipes Fabricius, 1792 & & 7 & 7 \\
\hline 89 & Hep.fulg & Helophorus fulgidicollis Motschuslky, 1860 & & 5 & 5 \\
\hline 90 & Hep.illu & Helophorus illustris Sharp, 1916 & & 6 & 6 \\
\hline 91 & Hep.long & Helophorus longitarsis Wollaston, 1864 & & 4 & 5 \\
\hline 92 & Hep.seid & Helophorus seidlitzii Kuwert, 1885 & $\mathrm{G}$ & 3 & 4 \\
\hline 93 & Geo.grcr & Georissus gr. crenulatus (Rossi, 1794) & & 6 & 6 \\
\hline 94 & Hch.flav & Hydrochus flavipennis Küster, 1852 & & 7 & 7 \\
\hline 95 & Hch.gran & Hydrochus grandicollis Kiesenwetter, 1870 & & 4 & 5 \\
\hline 96 & Hch.iber & $\begin{array}{l}\text { Hydrochus ibericus Valladares, } \\
\text { Díaz-Pazos \& Delgado, } 1999\end{array}$ & $\mathrm{~S}$ & 5 & 6 \\
\hline 97 & Hch.niti & Hydrochus nitidicollis Mulsant, 1844 & & 5 & 5 \\
\hline 98 & Hch.noor & $\begin{array}{l}\text { Hydrochus nooreinus Henegouven } \\
\text { \& Sáinz-Cantero, } 1992\end{array}$ & $\mathrm{~S}$ & 4 & 5 \\
\hline 99 & Ber.affi & Berosus affinis Brullé, 1835 & & 3 & 3 \\
\hline
\end{tabular}


Anexo 1. (Continuación.)

\begin{tabular}{|c|c|c|c|c|c|}
\hline 100 & Ber.hisp & Berosus hispanicus Küster, 1847 & & 1 & 2 \\
\hline 101 & Ber.fulv & Berosus fulvus Kuwert, 1888 & & 5 & 5 \\
\hline 102 & Ber.gutt & Berosus guttalis Rey, 1883 & & 5 & 5 \\
\hline 103 & Hem.guig & Hemisphaera guignoti Shalberg, 1900 & & 7 & 7 \\
\hline 104 & Cha.semi & Chaetarthria seminulum seminulum (Herbst, 179 & & 5 & 5 \\
\hline 105 & Cha.simi & Chaetarthria similis Wollaston, 1864 & & 5 & 5 \\
\hline 106 & Par.aene & Paracymus aeneus (Germar, 1824) & & 4 & 5 \\
\hline 107 & Par.rela & Paracymus relaxus Rey, $1884 *$ & & & \\
\hline 108 & Par.scut & Paracymus scutellaris (Rosenhauer, 1856) & & 7 & 7 \\
\hline 109 & Ana.bipu & Anacaena bipustulata (Marsham, 1802) & & 2 & 3 \\
\hline 110 & Ana.glob & Anacaena globulus (Paykull, 1798) & & 4 & 4 \\
\hline 111 & Ana.lute & Anacaena lutescens (Stephens, 1829) & & 3 & 4 \\
\hline 112 & Ana.limb & Anacaena limbata (Fabricius, 1792) & & 3 & 3 \\
\hline 113 & Lab.inte & $\begin{array}{l}\text { Laccobius gracillis intermittens } \\
\text { Kiesenwetter in Heyden, } 1870\end{array}$ & & 2 & 2 \\
\hline 114 & Lab.atro & Laccobius atrocephalus Reitter, 1872 & & 5 & 6 \\
\hline 115 & Lab.bipu & Laccobius bipunctatus (Fabricius, 1775) & & 4 & 5 \\
\hline 116 & Lab.hisp & Laccobius hispanicus Gentili, 1974 & & 2 & 2 \\
\hline 117 & Lab.mora & Laccobius moraguesi Régimbart, 1898 & & 2 & 3 \\
\hline 118 & Lab.neap & Laccobius neapolitanus Rottenberg, 1874 & & 4 & 4 \\
\hline 119 & Lab.obsc & Laccobius obscuratus Rottenberg, 1874 & & 3 & 4 \\
\hline 120 & Lab.sinu & Laccobius sinuatus Motschulsky, 1849 & & 2 & 3 \\
\hline 121 & Lab.yten & Laccobius ytenensis Sharp, 1910 & & 4 & 5 \\
\hline 122 & Hec.livi & Helochares lividus (Forster, 1771) & & 1 & 1 \\
\hline 123 & Eno.mela & Enochrus melanocephalus (Olivier, 1792)* & & & \\
\hline 124 & Eno.ater & Enochrus ater (Kuwert, 1888) & & 5 & 5 \\
\hline 125 & Eno.bico & Enochrus bicolor (Fabricius, 1792) & & 2 & 3 \\
\hline 126 & Eno.falc & Enochrus falcarius Hebauer, 1991 & & 4 & 4 \\
\hline 127 & Eno.fusc & Enochrus fuscipennis (Thomson, 1884) & & 7 & 7 \\
\hline 128 & Eno.halo & Enochrus halophilus (Bedel, 1878) & & 7 & 7 \\
\hline 129 & Eno.poli & Enochrus politus Küster, 1849 & & 1 & 2 \\
\hline 130 & Eno.salo & Enochrus salomonis (Sahlberg, 1900) & & 5 & 5 \\
\hline 131 & Eno.segm & Enochrus segmentinotatus (Kuwert, 1888) & & 7 & 7 \\
\hline 132 & Eno.test & Enochrus testaceus (Fabricius, 1801)** & & 6 & 6 \\
\hline 133 & Hbs.conv & Hydrobius convexus Brullé, 1835 & & 6 & 7 \\
\hline 134 & Hbs.fusc & Hydrobius fuscipes (Linnaeus, 1758) & & 5 & 6 \\
\hline 135 & Hdc.flav & Hydrochara flavipes (Steven, 1808) & & 6 & 6 \\
\hline 136 & Hph.pist & Hydrophilus pistaceus (Castelnau, 1840) & & 2 & 3 \\
\hline 137 & Coo.hisp & Coelostoma hispanicum (Küster, 1848) & & 2 & 2 \\
\hline 138 & Hdn.exas & Hydraena exasperata Orchymont, 1935 & $\mathrm{G}$ & 4 & 5 \\
\hline 139 & Hdn.manf & Hydraena manfredjaechi Delgado \& Soler, 1991 & $\mathrm{E}$ & 5 & 5 \\
\hline 140 & Hdn.afus & Hydraena afussa Orchymont, 1936 & $\mathrm{G}$ & 5 & 5 \\
\hline 141 & Hdn.bisu & Hydraena bisulcata Rey, 1884 & & 7 & 7 \\
\hline 142 & Hdn.boli & Hydraena bolivari Orchymont, 1936 & $\mathrm{~S}$ & 6 & 7 \\
\hline 143 & Hdn.brac & Hydraena brachymera Orchymont, 1936** & $\mathrm{NE}$ & 6 & 6 \\
\hline 144 & Hdn.capt & Hydraena capta Orchymont, 1936 & & 3 & 4 \\
\hline 145 & Hdn.carb & Hydraena carbonaria Kiesenwetter, 1849 & $\mathrm{NE}$ & 3 & 4 \\
\hline 146 & Hdn.clar & Hydraena rufipennis BoscàBerga, 1836 & & 2 & 3 \\
\hline 147 & Hdn.cord & Hydraena cordata Schaufuss, 1833 & & 4 & 5 \\
\hline 148 & Hdn.flav & Hydraena flavipes Stephens, 1829 & & 4 & 5 \\
\hline 149 & Hdn.meca & Hydraena mecai Millán \& Aguilera 2000 & $\mathrm{E}$ & 7 & 7 \\
\hline 150 & Hdn.pygm & Hydraena pygmaea Waterhouse, 1833 & & 6 & 6 \\
\hline 151 & Hdn.quil & $\begin{array}{l}\text { Hydraena quilisi Lagar, } \\
\text { Fresneda \& Hernando, } 1987\end{array}$ & $\mathrm{NE}$ & 4 & 5 \\
\hline
\end{tabular}


Anexo 1. (Continuación.)

\begin{tabular}{|c|c|c|c|c|c|}
\hline 152 & Hdn.ripa & Hydraena riparia Kugelann, 1794 & & 6 & 7 \\
\hline 153 & Hdn.serv & Hydraena servilla Orchymont, 1936 & $\mathrm{~S}$ & 4 & 4 \\
\hline 154 & Hdn.unca & Hydraena unca Valladares, 1989 & $\mathrm{G}$ & 7 & 7 \\
\hline 155 & Hdn.hern & Hydraena hernandoi Fresneda \& Lagar, 1990 & & 4 & 4 \\
\hline 156 & Hdn.test & Hydraena testacea Curtis, $1830 * *$ & & 5 & 6 \\
\hline 157 & Lib.cord & Limnebius cordobanus Orchymont, 1938 & $\mathrm{G}$ & 4 & 4 \\
\hline 158 & Lib.furc & Limnebius furcatus Baudi, 1872 & & 5 & 5 \\
\hline 159 & Lib.gerh & Limnebius gerhardti Heyden, 1870 & $\mathrm{G}$ & 7 & 7 \\
\hline 160 & Lib.hisp & Limnebius hispanicus Orchymont, 1941 & $\mathrm{~S}$ & 6 & 6 \\
\hline 161 & Lib.maur & Limnebius maurus Balfour-Browne, 1978 & & 2 & 3 \\
\hline 162 & Lib.mill & Limnebius millani Ribera \& Hernando, 1998 & $\mathrm{E}$ & 6 & 6 \\
\hline 163 & Lib.oblo & Limnebius oblongus Rey, 1883 & & 4 & 4 \\
\hline 164 & Lib.papp & Limnebius papposus Mulsant, 1844 & & 6 & 7 \\
\hline 165 & Lib.trun & Limnebius truncatellus (Thunberg, 1794) & & 6 & 6 \\
\hline 166 & Eni.exsc & Enicocerus exsculptus Germar, 1824 & & 5 & 5 \\
\hline 167 & Cal.quad & Calobius quadricollis (Mulsant, 1844) & & 6 & 7 \\
\hline 168 & Och.aene & Ochthebius aeneus Stephens, 1835 & & 5 & 5 \\
\hline 169 & Och.bell & Ochthebius bellieri Kuwert, 1887 & $\mathrm{~S}$ & 6 & 6 \\
\hline 170 & Och.bonn & Ochthebius bonnairei Guillebau, 1896 & & 3 & 4 \\
\hline 171 & Och.dila & Ochthebius dilatatus Stephens, 1829 & & 3 & 4 \\
\hline 172 & Och.iren & Ochthebius irenae Ribera \& Millán, 1998 & $\mathrm{~S}$ & 7 & 7 \\
\hline 173 & Och.macu & Ochthebius maculatus Reiche, 1872 & & 4 & 4 \\
\hline 174 & Och.subi & Ochthebius subinteger Mulsant \& Rey, 1861 & & 6 & 7 \\
\hline 175 & Och.alba & Ochthebius albacetinus Ferro, 1984 & $\mathrm{E}$ & 6 & 6 \\
\hline 176 & Och.auro & Ochthebius auropallens Fairmaire, 1879 & & 3 & 3 \\
\hline 177 & Och.bifo & Ochthebius bifoveolatus Waltl, 1835 & & 6 & 6 \\
\hline 178 & Och.corr & Ochthebius corrugatus Rosenhauer, 1856 & & 4 & 4 \\
\hline 179 & Och.cupr & Ochthebius cuprescens Guillenbau, 1893 & & 3 & 3 \\
\hline 180 & Och.delg & Ochthebius delgadoi Jäch, 1994 & $\mathrm{G}$ & 2 & 3 \\
\hline 181 & Och.dent & Ochthebius dentifer Rey, 1885 & & 7 & 7 \\
\hline 182 & Och.diff & Ochthebius difficilis Mulsant, 1844 & & 4 & 4 \\
\hline 183 & Och.glab & Ochthebius glaber Montes \& Soler, 1988 & $\mathrm{~S}$ & 5 & 6 \\
\hline 184 & Och.gran & Ochthebius grandipennis Fairmaire, 1879 & & 4 & 5 \\
\hline 185 & Och.marg & Ochthebius marginalis Rey, 1886 & $\mathrm{NE}$ & 6 & 7 \\
\hline 186 & Och.mari & Ochthebius marinus (Paykull, 1798) & & 3 & 4 \\
\hline 187 & Och.medi & Ochthebius mediterraneus Ieniestea, 1988 & & 4 & 4 \\
\hline 188 & Och.meta & Ochthebius metallescens Rosenhauer, 1847 & & 5 & 5 \\
\hline 189 & Och.mont & Ochthebius montesi Ferro, 1984 & $\mathrm{~S}$ & 5 & 5 \\
\hline 190 & Och.nanu & Ochthebius nanus Stephens, 1829 & & 4 & 4 \\
\hline 191 & Och.nota & Ochthebius notabilis Rosenhauer, 1856 & & 6 & 6 \\
\hline 192 & Och.quav & Ochthebius quadrifoveolatus Wollaston, 1854 & & 2 & 3 \\
\hline 193 & Och.semi & Ochthebius semotus d'Orchymont, 1942** & & 6 & 6 \\
\hline 194 & Och.subp & Ochthebius subpictus Wollaston, 1857 & & 6 & 6 \\
\hline 195 & Och.taca & Ochthebius tacapasensis baeticus Ferro, 1984 & & 3 & 4 \\
\hline 196 & Och.tudm & Ochthebius tudmirensis Jäch, 1997 & $\mathrm{~S}$ & 3 & 4 \\
\hline 197 & Och.vire & Ochthebius viridis 2 sensu Jäch, 1991 & & 4 & 4 \\
\hline 198 & Elo.sp & Elodes sp. & & 6 & 6 \\
\hline 199 & Hcp.sp & Hydrocyphon sp. & & 4 & 4 \\
\hline 200 & Pot.acum & Potamophilus acuminatus (Fabricius, 1792) & & 5 & 5 \\
\hline 201 & Ste.cana & Stenelmis canaliculata (Gyllenhal, 1808) & & 6 & 7 \\
\hline 202 & Elm.aene & Elmis aenea (Müller, 1806) & & 3 & 3 \\
\hline 203 & Elm.maug & Elmis maugetii maugetii Latreille, 1798 & & 2 & 3 \\
\hline 204 & Elm.riol & Elmis rioloides (Kuwert, 1890) & & 5 & 5 \\
\hline
\end{tabular}


Anexo 1. (Continuación.)

\begin{tabular}{|c|c|c|c|c|}
\hline 205 & Eso.para & Esolus parallelepipedus (Müller, 1806) & 3 & 4 \\
\hline 206 & Eso.pygm & Esolus pygmaeus (Müller, 1806) & 6 & 6 \\
\hline 207 & Oul.trog & Oulimnius troglodytes (Gyllenhal, 1827) & 2 & 3 \\
\hline 208 & Oul.tube & Oulimnius tuberculatus tuberculatus (Müller, 1806)** & 7 & 7 \\
\hline 209 & Oul.pere & Oulimnius tuberculatus perezi Sharp, $1872 \quad$ NE & 4 & 4 \\
\hline 210 & Lin.inte & Limnius intermedius Fairmaire, 1881 & 2 & 3 \\
\hline 211 & Lin.opac & Limnius opacus Müller, 1806 & 2 & 3 \\
\hline 212 & Lin.volc & Limnius volckmari (Panzer, 1793) & 3 & 4 \\
\hline 213 & Nor.nite & Normandia nitens (Müller, 1817) & 3 & 4 \\
\hline 214 & Nor.soda & Normandia sodalis (Erichson, 1847) & 2 & 3 \\
\hline 215 & Rio.cupr & Riolus cupreus (Müller, 1806) & 2 & 3 \\
\hline 216 & Rio.illi & Riolus illiesi Steffan, 1958 & 4 & 4 \\
\hline 217 & Rio.subv & Riolus subviolaceus (Müller, 1817) & 5 & 5 \\
\hline 218 & Pom.subs & Pomatinus substriatus (Müller, 1806) & 2 & 3 \\
\hline 219 & Dry.algi & Dryops algiricus (Lucas, 1849) & 7 & 7 \\
\hline 220 & Dry.grac & Dryops gracilis (Karsch, 1881) & 1 & 1 \\
\hline 221 & Dry.luri & Dryops luridus (Erichson, 1847) & 5 & 5 \\
\hline 222 & Dry.lutu & Dryops lutulentus (Erichson, 1847) & 4 & 5 \\
\hline 223 & Dry.sulc & Dryops sulcipennis (Costa, 1883) & 4 & 4 \\
\hline 224 & Het.flex & Heterocerus flexuosus (Stephens, 1828) & 7 & 7 \\
\hline 225 & Aug.mari & Augyles maritimus (Guérin-Méneville, 1844) & 6 & 6 \\
\hline 226 & Don.anda & Donacia andalusiaca Kraatz, $1869 *$ & & \\
\hline 227 & Don.aqua & Donacia aquatica (Linnaeus, 1758) * & & \\
\hline 228 & Plr.seri & Plateumaris seriacea (Linnaeus, 1758) * & & \\
\hline 229 & Bag.sp & Bagous sp. & 6 & 6 \\
\hline 230 & Lic.sp & Limnichus sp. & 6 & 6 \\
\hline
\end{tabular}


Anexo 2. Valores de IC para las cuadrícula UTM 10 x $10 \mathrm{Km}$. muestreadas. S: riqueza de especies. IC scores of the UTM $10 \times 10 \mathrm{Km}$. squares sampled. S: species richness.

\begin{tabular}{|c|c|c|c|c|c|c|c|c|}
\hline Cuadrícula & $\mathbf{S}$ & IC & Cuadrícula & $\mathbf{S}$ & IC & Cuadrícula & $\mathbf{S}$ & IC \\
\hline WH5.6 & 122 & 940.93 & XG2.9 & 30 & 20.41 & XH1.2 & 17 & 6.40 \\
\hline WH3.3 & 74 & 249.48 & WH6.2 & 21 & 19.51 & XH5.1 & 16 & 6.36 \\
\hline WH4.6 & 72 & 240.76 & XH6.0 & 21 & 18.94 & WH7.6 & 14 & 6.23 \\
\hline WH3.2 & 56 & 160.18 & WH8.6 & 23 & 18.56 & XH9.1 & 13 & 6.07 \\
\hline WH5.7 & 55 & 149.79 & WH8.2 & 19 & 18.08 & XH4.6 & 11 & 5.74 \\
\hline WH4.4 & 61 & 138.58 & WH7.3 & 20 & 17.20 & XH2.3 & 16 & 5.63 \\
\hline WH5.4 & 52 & 128.58 & XH4.2 & 27 & 17.02 & XG4.7 & 2 & 5.60 \\
\hline WH4.5 & 43 & 110.73 & XH9.3 & 9 & 16.94 & WG9.8 & 15 & 5.48 \\
\hline XH1.6 & 52 & 106.08 & YG0.6 & 8 & 16.39 & XG3.6 & 13 & 5.33 \\
\hline WH6.7 & 56 & 104.33 & XH3.9 & 14 & 16.07 & XH4.1 & 14 & 5.29 \\
\hline WH7.2 & 51 & 87.04 & WH7.5 & 25 & 15.77 & XH 2.5 & 16 & 5.09 \\
\hline XJ2.0 & 41 & 79.65 & XH1.7 & 11 & 15.75 & XG1.7 & 14 & 5.02 \\
\hline $\mathrm{XJ} 3.0$ & 35 & 68.66 & XH5.0 & 14 & 15.67 & WH7.4 & 7 & 4.88 \\
\hline WH5.5 & 46 & 68.58 & YH0.2 & 17 & 14.48 & XG4.5 & 15 & 4.81 \\
\hline XH6.2 & 45 & 67.83 & XG2.5 & 17 & 14.41 & WH9.6 & 8 & 4.66 \\
\hline WH9.1 & 44 & 63.99 & WH8.1 & 20 & 13.37 & XG3.9 & 10 & 4.63 \\
\hline WH3.1 & 37 & 61.51 & XH9.2 & 8 & 13.26 & XH2.2 & 9 & 4.61 \\
\hline WH9.3 & 42 & 52.30 & YH0.1 & 17 & 12.66 & XH0.6 & 11 & 4.41 \\
\hline WH6.5 & 43 & 50.36 & WH4.2 & 20 & 12.26 & XH1.5 & 6 & 4.35 \\
\hline XH1.3 & 43 & 46.49 & XG6.9 & 20 & 11.98 & XH2.1 & 11 & 4.20 \\
\hline XH3.1 & 45 & 44.18 & XG8.7 & 17 & 11.72 & XG9.7 & 3 & 4.16 \\
\hline XG3.5 & 33 & 44.05 & WH7.7 & 15 & 11.63 & XH7.3 & 7 & 3.31 \\
\hline XH4.3 & 42 & 43.53 & XH5.2 & 22 & 11.13 & WH8.3 & 5 & 3.22 \\
\hline WH4.1 & 33 & 43.25 & XH1.4 & 16 & 10.85 & XG0.9 & 4 & 2.28 \\
\hline WH6.6 & 39 & 39.05 & XG8.9 & 2 & 10.18 & XH3.2 & 3 & 2.25 \\
\hline XH1.0 & 38 & 36.84 & XG0.6 & 14 & 10.11 & XH4.0 & 8 & 2.17 \\
\hline WH4.3 & 36 & 32.04 & XH3.4 & 14 & 9.98 & WG8.8 & 4 & 2.01 \\
\hline XG1.8 & 26 & 30.83 & WH6.4 & 17 & 9.03 & XH0.4 & 4 & 2.01 \\
\hline XH3.3 & 38 & 30.57 & WH9.5 & 9 & 8.25 & XG7.6 & 1 & 2.01 \\
\hline WG9.7 & 37 & 30.37 & WH2.1 & 7 & 7.75 & XH2.6 & 3 & 1.91 \\
\hline WH5.2 & 31 & 27.99 & WG8.7 & 15 & 7.62 & WH8.0 & 3 & 1.91 \\
\hline XG9.6 & 16 & 24.17 & XG5.5 & 4 & 7.50 & XH6.1 & 6 & 1.64 \\
\hline XH2.0 & 32 & 24.03 & XH8.1 & 14 & 7.43 & XH0.2 & 4 & 1.61 \\
\hline XH0.8 & 20 & 23.82 & WH6.3 & 15 & 7.35 & XG4.9 & 1 & 1 \\
\hline WH5.3 & 29 & 23.12 & XH7.0 & 14 & 7.30 & WH9.4 & 1 & 1 \\
\hline XG9.8 & 14 & 22.77 & WG9.9 & 10 & 7.02 & XH0.5 & 2 & 0.51 \\
\hline XG4.6 & 30 & 21.99 & XH0.1 & 12 & 6.98 & XH7.1 & 1 & 0.5 \\
\hline WH8.5 & 26 & 21.87 & XH1.1 & 17 & 6.74 & XG6.6 & 1 & 0.5 \\
\hline XH7.2 & 30 & 21.74 & XG0.7 & 4 & 6.56 & & & \\
\hline
\end{tabular}


